\title{
САМОПЕРЕСЕЧЕНИЯ В ПАРАМЕТРИЗОВАННЫХ САМОПОДОБНЫХ МНОЖЕСТВАХ ПРИ СДВИГАХ И РАСТЯЖЕНИЯХ КОПИЙ ${ }^{1}$
}

\section{К. Г. Камалутдинов}

\begin{abstract}
В работе рассматривается проблема пересечения $F_{i}\left(K_{t}\right) \cap F_{j}^{t}\left(K_{t}\right)$ пар различных копий самоподобного множества $K_{t}$, порожденного системой $\mathcal{F}_{t}=\left\{F_{1}, \ldots, F_{m}\right\}$ сжимающих подобий в $\mathbb{R}^{n}$, в которой одно отображение $F_{j}^{t}$ зависит от вещественного или векторного параметра $t$. Рассмотрены два случая: параметр $t \in \mathbb{R}^{n}$ задает сдвиг отображения $F_{j}^{t}(x)=G(x)+t$ и параметр $t \in(a, b)$ задает коэффициент подобия отображения $F_{j}^{t}(x)=t G(x)+h$, где $0<a<b<1$, а $G$ - изометрия в $\mathbb{R}^{n}$. Мы накладываем некоторые ограничения на коэффициенты подобия отображений системы $\mathcal{F}_{t}$ и требуем, чтобы размерность подобия системы была не больше некоторого $s$. Для таких систем доказано, что хаусдорфова размерность множества тех параметров $t$, при которых пересечение $F_{i}\left(K_{t}\right) \cap F_{j}^{t}\left(K_{t}\right)$ непусто, не превосходит $2 s$. Полученные результаты применены к проблеме проверки строгого условия отделимости (SSC) для системы $\mathcal{F}_{\tau}=\left\{F_{1}^{\tau}, \ldots, F_{m}^{\tau}\right\}$ сжимающих подобий, зависящей от набора параметров $\tau=\left(t_{1}, \ldots, t_{m}\right)$. Рассмотрены два случая: $\tau$ - набор сдвигов отображений $F_{i}^{\tau}(x)=G_{i}(x)+t_{i}, t_{i} \in \mathbb{R}^{n}$, и $\tau$ - набор коэффициентов подобия отображений $F_{i}^{\tau}(x)=t_{i} G_{i}(x)+h_{i}, t_{i} \stackrel{i}{\in}(a, b)$, где $0<a<b<1$, а все $G_{i}$ - изометрии в $\mathbb{R}^{n}$. В обоих случаях мы находим достаточные условия, при которых система $\mathcal{F}_{\tau}$ удовлетворяет SSC для почти всех значений параметров $\tau$. Кроме того, рассмотрена более простая проблема пересечения $A \cap f_{t}(B)$ для пары компактных подмножеств $A, B$ пространства $\mathbb{R}^{n}$. Рассмотрены два случая: $f_{t}(B)=B+t$ для $t \in \mathbb{R}^{n}$, и $f_{t}(B)=t B$ для $t \in \mathbb{R}$, где замыкание $B$ не содержит 0. В обоих случаях доказано, что хаусдорфова размерность множества тех параметров $t$, при которых пересечение $A \cap f_{t}(B)$ непусто, не превосходит $\operatorname{dim}_{H}(A \times B)$. Как следствие, при достаточно малой размерности произведения $A \times B$ в обоих случаях гарантировано пустое пересечение $A \cap f_{t}(B)$ для почти всех значений параметра $t$.
\end{abstract}

Ключевые слова: самоподобный фрактал, общее положение, строгое условие отделимости, размерность Хаусдорфа.

K. G. Kamalutdinov. Self-intersections in parametrized self-similar sets under translations and extensions of copies.

We study the problem of pairwise intersections $F_{i}\left(K_{t}\right) \cap F_{j}^{t}\left(K_{t}\right)$ of different copies of a self-similar set $K_{t}$ generated by a system $\mathcal{F}_{t}=\left\{F_{1}, \ldots, F_{m}\right\}$ of contracting similarities in $\mathbb{R}^{n}$, where one mapping $F_{j}^{t}$ depends on a real or vector parameter $t$. Two cases are considered: the parameter $t \in \mathbb{R}^{n}$ specifies a translation of a mapping $F_{j}^{t}(x)=G(x)+t$, and the parameter $t \in(a, b)$ is the similarity coefficient of a mapping $F_{j}^{t}(x)=t G(x)+h$, where $0<a<b<1$ and $G$ is an isometry of $\mathbb{R}^{n}$. We impose some constraints on the similarity coefficients of mappings of the system $\mathcal{F}_{t}$ and require that the similarity dimension of the system does not exceed some number $s$. For such systems it is proved that the Hausdorff dimension of the set of parameters $t$ for which the intersection $F_{i}\left(K_{t}\right) \cap F_{j}^{t}\left(K_{t}\right)$ is nonempty does not exceed $2 s$. The obtained results are applied to the problem of checking the strong separation condition for a system $\mathcal{F}_{\tau}=\left\{F_{1}^{\tau}, \ldots, F_{m}^{\tau}\right\}$ of contraction similarities depending on a parameter vector $\tau=\left(t_{1}, \ldots, t_{m}\right)$. Two cases are considered: $\tau$ is a vector of translations of mappings $F_{i}^{\tau}(x)=G_{i}(x)+t_{i}, t_{i} \in \mathbb{R}^{n}$, and $\tau$ is a vector of similarity coefficients of mappings $F_{i}^{\tau}(x)=t_{i} G_{i}(x)+h_{i}$, $t_{i} \in(a, b)$, where $0<a<b<1$ and all $G_{i}$ are isometries in $\mathbb{R}^{n}$. In both cases we find sufficient conditions for the system $\mathcal{F}_{\tau}$ to satisfy the strong separation condition for almost all values of $\tau$. We also consider the easier problem of the intersection $A \cap f_{t}(B)$ for a pair of compact sets $A$ and $B$ in the space $\mathbb{R}^{n}$. Two cases are considered: $f_{t}(B)=B+t$ for $t \in \mathbb{R}^{n}$, and $f_{t}(B)=t B$ for $t \in \mathbb{R}$, where the closure of $B$ does not contain the origin. In both cases it is proved that the Hausdorff dimension of the set of parameters $t$ for which the intersection $A \cap f_{t}(B)$ is nonempty does not exceed $\operatorname{dim}_{H}(A \times B)$. Consequently, when the dimension of the product $A \times B$ is small enough, the empty intersection $A \cap f_{t}(B)$ is guaranteed for almost all values of $t$ in both cases.

Keywords: self-similar fractal, general position, strong separation condition, Hausdorff dimension.

MSC: 28A78, 28A 80

DOI: $10.21538 / 0134-4889-2019-25-2-116-124$

${ }^{1}$ Работа выполнена при поддержке РФФИ (проекты 19-01-00569, 18-501-51021). 


\section{Введение}

Отображение $F: \mathbb{R}^{n} \rightarrow \mathbb{R}^{n}$ называется подобием, если существует константа $p>0$, называемая коэффициентом подобия отображения $F$, такая, что $\|F(x)-F(y)\|=p\|x-y\|$ для всех $x, y \in \mathbb{R}^{n}$. Подобие $F$ называется сжсимающим, если $p<1$. Пусть $\mathcal{F}=\left\{F_{1}, \ldots, F_{m}\right\}-$ система сжимающих подобий в $\mathbb{R}^{n}$. Непустой компакт $K$ такой, что $K=\bigcup_{i=1}^{m} F_{i}(K)$, называется самоподобным множеством, порожденным системой $\mathcal{F}$, или аттрактором этой системы. Такой $K$ существует и единственен по теореме Хатчинсона [1]. Множества $F_{i}(K), 1 \leq i \leq m$, называются копиями множества $K$.

Пусть $\mathcal{F}_{t}=\left\{F_{1}^{t}, \ldots, F_{m}^{t}\right\}$ - система сжимающих подобий в $\mathbb{R}^{n}$ с аттрактором $K_{t}$, зависящая от вещественного или векторного параметра $t \in D$. Зафиксируем различные индексы $i, j \in$ $\{1, \ldots, m\}$. Возникает вопрос, насколько велико множество $\Delta$ тех параметров, при которых пересечение $F_{i}^{t}\left(K_{t}\right) \cap F_{j}^{t}\left(K_{t}\right)$ копий аттрактора $K_{t}$ непусто?

В данной работе мы рассмотрим два вида таких систем, где параметр $t$ будет играть роль вектора сдвига или коэффициента подобия одного из отображений $F_{j}^{t}$ системы $\mathcal{F}_{t}$, в то время как другие отображения не будут зависеть от параметра $t$.

Кроме того, мы рассмотрим более простую задачу оценки размерности множества параметров $\Delta=\left\{t \in D: A \cap f_{t}(B) \neq \varnothing\right\}$ для пары произвольных множеств $A$ и $B$ в $\mathbb{R}^{n}$, где одно из них подвергается сдвигам $\left(f_{t}(B)=B+t\right)$ или гомотетиям $\left(f_{t}(B)=t B\right)$.

Во всех этих случаях для рассматриваемых семейств $\left(A_{t}, B_{t}\right)$ пар множеств, зависящих от параметра $t \in D$, исключительными параметрами будем называть те $t \in D$, при которых пересечение $A_{t} \cap B_{t}$ непусто.

Вопрос о размерности пересечения $A \cap f_{t}(B)$ пар множеств, где одно из них подвергается преобразованиям $f_{t}$ таким, как сдвиги, повороты или растяжения, рассматривался рядом авторов. Так, Марстранд [2] рассматривал пересечения подмножеств плоскости с прямыми, а Маттила [3] и Фалконер [4] - пересечения пар борелевских и суслинских подмножеств евклидова пространства. Однако если преобразованиям $f_{t}$ подвергается одно или несколько сжимающих подобий в системе $\mathcal{F}_{t}=\left\{F_{1}^{t}, \ldots, F_{m}^{t}\right\}$, например, $F_{j}^{t}=f_{t} \circ G$, то для пересечения $F_{i}^{t}\left(K_{t}\right) \cap F_{j}^{t}\left(K_{t}\right)$ копий аттрактора $K_{t}$ системы $\mathcal{F}_{t}$ ситуация усложняется.

Размерностью подобия системы $\mathcal{F}=\left\{F_{1}, \ldots, F_{m}\right\}$ называется решение $s$ уравнения Морана $\sum_{i=1}^{m} p_{i}^{s}=1$, где $p_{i}-$ коэффициенты подобия отображений $F_{i}$. Симоном и Полликоттом [5] был предложен подход, основанный на условии трансверсальности и методах теории потенциала. Этот подход может гарантировать совпадение хаусдорфовой размерности аттрактора $K_{t}$ и размерности подобия системы $\mathcal{F}_{t}$ для почти всех значений параметра $t$. Однако при этом он не гарантирует того, что пересечения $F_{i}^{t}\left(K_{t}\right) \cap F_{j}^{t}\left(K_{t}\right)$ пусты.

Для решения поставленной задачи мы используем полученную нами теорему об общем положении [6, Theorem 14]. Наш метод позволяет оценивать хаусдорфову размерность множества исключительных параметров $t$ и находить условия, при которых пересечения $F_{i}^{t}\left(K_{t}\right) \cap F_{j}^{t}\left(K_{t}\right)$ пусты для почти всех значений параметра $t$.

Мы также покажем, как решение поставленной нами задачи может быть использовано для проверки строгого условия отделимости. Система $\mathcal{F}=\left\{F_{1}, \ldots, F_{m}\right\}$ cтрого отделима, или удовлетворяет строгому условию отделимости (SSC - strong separation condition), если $F_{i}(K) \cap F_{j}(K)=\varnothing$ для всех $1 \leq i<j \leq m$. Хорошо известно, что если система $\mathcal{F}$ удовлетворяет $\mathrm{SSC}$, то размерность ее аттрактора $K$ совпадает с размерностью подобия системы $\mathcal{F}$.

Пусть система $\mathcal{F}_{\tau}=\left\{F_{1}^{\tau}, \ldots, F_{m}^{\tau}\right\}$ сжимающих подобий в $\mathbb{R}^{n}$ зависит от набора параметров $\tau$. Мы рассмотрим два случая, в которых набор параметров $\tau=\left(t_{1}, \ldots, t_{m}\right)$ задает сдвиги отображений $F_{i}^{\tau}(x)=G_{i}(x)+t_{i}, t_{i} \in \mathbb{R}^{n}, 1 \leq i \leq m$, либо коэффициенты подобия отображений $F_{i}^{\tau}(x)=t_{i} G_{i}(x)+h_{i}, t_{i} \in(a, b)$, где $0<a<b<1$, а все $G_{i}$ - изометрии пространства $\mathbb{R}^{n}$. В обоих случаях мы находим достаточные условия, при которых система $\mathcal{F}_{\tau}$ удовлетворяет условию SSC для почти всех значений параметров $\tau$. 


\section{1. Определения и обозначения}

Везде, где рассматривается система $\mathcal{F}=\left\{F_{1}, \ldots, F_{m}\right\}$ сжимающих подобий (возможно, зависящая от параметра), мы будем использовать следующие обозначения:

$I=\{1, \ldots, m\}-$ множество индексов;

$I^{n}$ - множество всех слов $\boldsymbol{i}=i_{1} \ldots i_{n}$ длины $n$ в алфавите $I$, называемых мультииндексами;

$I^{*}=\bigcup_{n=1}^{\infty} I^{n}-$ множество всех мультииндексов;

$I^{\infty}=\left\{\alpha=\alpha_{1} \alpha_{2} \ldots: \alpha_{i} \in I\right\}-$ индексное пространство;

для последовательностей $\alpha, \beta \in I^{\infty} \quad \alpha \wedge \beta$ - их наибольший общий начальный отрезок;

для мультииндекса $\boldsymbol{i}=i_{1} \ldots i_{k} \in I^{k} \quad F_{\boldsymbol{i}}=F_{i_{1}} \circ \ldots \circ F_{i_{k}}$ - композиция отображений системы $\mathcal{F}$; для набора чисел $\left(p_{1}, \ldots, p_{m}\right)$ длины $m$ и мультииндекса $\boldsymbol{i}=i_{1} \ldots i_{k} \quad p_{\boldsymbol{i}}=p_{i_{1}} \cdot \ldots \cdot p_{i_{k}}$.

Отображение $\pi: I^{\infty} \rightarrow K, \pi: \alpha \mapsto \lim _{n \rightarrow \infty} F_{\alpha_{1} \ldots \alpha_{n}}(0)$ называется индексной параметризацией аттрактора $K$.

Через $\operatorname{dim}_{H} X$ будем обозначать хаусдорфову размерность множества $X$, а через $\operatorname{dim}_{S} \mathcal{F}-$ размерность подобия системы $\mathcal{F}$.

\section{2. Теорема об общем положении}

Сформулируем теорему об общем положении [6, Theorem 14] в следующем упрощенном варианте.

Теорема 1. Пусть $\left(L_{1}, \sigma_{1}\right),\left(L_{2}, \sigma_{2}\right)$ - компактные метрические пространства, $D \subseteq \mathbb{R}^{m}$, $\varphi_{1}: D \times L_{1} \rightarrow \mathbb{R}^{n}$ u $\varphi_{2}: D \times L_{2} \rightarrow \mathbb{R}^{n}$ - непрерывные отображения, удовлетворяющие условиям:

(a) для некоторого $C>0$ и всех $t \in D, x \in L_{1}, y \in L_{2} u i=1,2$

$$
\left\|\varphi_{i}(t, x)-\varphi_{i}(t, y)\right\| \leq C \cdot \sigma_{i}(x, y)
$$

(b) для некоторого $M>0$ и всех $\left(x_{1}, x_{2}\right) \in L_{1} \times L_{2}, t, t^{\prime} \in D$ функиия $\Phi\left(t, x_{1}, x_{2}\right)=$ $\varphi_{1}\left(t, x_{1}\right)-\varphi_{2}\left(t, x_{2}\right)$ удовлетворяет условию

$$
M \cdot\left\|t^{\prime}-t\right\| \leq\left\|\Phi\left(t^{\prime}, x_{1}, x_{2}\right)-\Phi\left(t, x_{1}, x_{2}\right)\right\| .
$$

Тогда множество $\Delta=\left\{t \in D: \varphi_{1}\left(t, L_{1}\right) \cap \varphi_{2}\left(t, L_{2}\right) \neq \varnothing\right\}$ замкнуто в $D u$

$$
\operatorname{dim}_{H} \Delta \leq \operatorname{dim}_{H}\left(L_{1} \times L_{2}\right) .
$$

Таким образом, если произведение $L_{1} \times L_{2}$ имеет достаточно малую размерность, а именно $\operatorname{dim}_{H}\left(L_{1} \times L_{2}\right)<d=\operatorname{dim}_{H} D$, то множества $\varphi\left(t, L_{1}\right)$ и $\psi\left(t, L_{2}\right)$ не пересекаются для почти всех $t \in D$ по $d$-мерной мере Хаусдорфа.

\section{3. Сдвиги и гомотетии одного множества}

Пусть дана пара компактных множеств $A, B \subseteq \mathbb{R}^{n}$ и к множеству $B$ применяется некоторое семейство преобразований $f_{t}$ пространства $\mathbb{R}^{n}$, зависящих от вещественного или векторного параметра $t \in D$. Нас интересует размерность множества исключительных параметров $\Delta=$ $\left\{t \in D: A \cap f_{t}(B) \neq \varnothing\right\}$. Ограничимся здесь рассмотрением двух случаев:

1) $f_{t}(B)=B+t$, где $B+t=\{b+t: b \in B\}$, т. е. множество $B$ сдвигается на векторный параметр $t \in \mathbb{R}^{n}$;

2) $f_{t}(B)=t B$, где $t B=\{t b: b \in B\}$, т. е. множество $B$ подвергается гомотетии с коэффициентами $t \in \mathbb{R}$.

Заметим, что случай 1 рассматривался в книге Фалконера [7, Exercise 8.4]. 


\section{1. Сдвиги}

Теорема 2. Пусть $A, B \subset \mathbb{R}^{n}-$ компакты, $\Delta=\left\{t \in \mathbb{R}^{n}: A \cap(B+t) \neq \varnothing\right\}$. Тогда множество $\Delta=\{a-b: a \in A, b \in B\}$ замкнуто $u \operatorname{dim}_{H} \Delta \leq \operatorname{dim}_{H}(A \times B)$.

Д о к а з а т е л ь с т в о. $A \cap(B+t) \neq \varnothing$ равносильно тому, что $a=b+t$ для некоторых $a \in A, b \in B$. Поэтому $\Delta=\{a-b: a \in A, b \in B\}$.

Рассмотрим отображения $\varphi: \mathbb{R}^{n} \times A \rightarrow A, \varphi(t, a)=a$, и $\psi: \mathbb{R}^{n} \times B \rightarrow \mathbb{R}^{n}, \psi(t, b)=b+t$. Пусть $\Phi(t, a, b)=\varphi(t, a)-\psi(t, b)$. Очевидно, что для $\varphi$ и $\psi$ выполняется условие (а) теоремы 1. Проверим условие (b)

$$
\varphi\left(t^{\prime}, a\right)-\varphi(t, a)=0 ; \quad \psi\left(t^{\prime}, b\right)-\psi(t, b)=t^{\prime}-t,
$$

откуда $\left\|\Phi\left(t^{\prime}, a, b\right)-\Phi(t, a, b)\right\|=\left\|t^{\prime}-t\right\|$. Применяя теорему 1 к функциям $\varphi$ и $\psi$, получаем, что множество $\Delta$ замкнуто и $\operatorname{dim}_{H} \Delta \leq \operatorname{dim}_{H}(A \times B)$.

Следствие 1. Пусть $A, B \subset \mathbb{R}^{n}-$ компакты, $D \subseteq \mathbb{R}^{n} . E c л и \operatorname{dim}_{H}(A \times B)<d=\operatorname{dim}_{H} D$, то $A \cap(B+t)=\varnothing$ для почти всех $t \in D$ по $d$-мерной мере Хаусдорба.

\section{2. Гомотетии}

Теорема 3. Пусть $A, B \subseteq \mathbb{R}^{n}-$ компакты и 0 не лежит в замыкании $B$. Тогда множество $\Delta=\{t \in \mathbb{R}: A \cap t B \neq \varnothing\}$ замкнуто и $\operatorname{dim}_{H} \Delta \leq \operatorname{dim}_{H}(A \times B)$.

Д о к а з а т е л ь с т в о. Рассмотрим отображения $\varphi: \mathbb{R} \times A \rightarrow A, \varphi(t, a)=a$, и $\psi: \mathbb{R} \times$ $B \rightarrow \mathbb{R}^{n}, \psi(t, b)=t b$. Пусть $\Phi(t, a, b)=\varphi(t, a)-\psi(t, b)$.

Очевидно, что для $\varphi$ и $\psi$ выполняется условие (a) теоремы 1 . Проверим условие (b). Пусть $r=\inf _{b \in B}\|b\|$. Поскольку 0 не лежит в замыкании $B$, имеем $r>0$. Поскольку

$$
\left\|\varphi\left(t^{\prime}, a\right)-\varphi(t, a)\right\|=0 ; \quad\left\|\psi\left(t^{\prime}, b\right)-\psi(t, b)\right\|=\|b\|\left|t^{\prime}-t\right| \geq r\left|t^{\prime}-t\right|,
$$

имеем $\left\|\Phi\left(t^{\prime}, a, b\right)-\Phi(t, a, b)\right\| \geq r\left|t^{\prime}-t\right|$. Применяя теорему 1 к функциям $\varphi$ и $\psi$, получаем, что множество $\Delta$ замкнуто и $\operatorname{dim}_{H} \Delta \leq \operatorname{dim}_{H}(A \times B)$.

Следствие 2. Пусть $A, B \subseteq \mathbb{R}^{n}-$ компакты, 0 не лежит в замыкании $B, u D \subseteq \mathbb{R}$. Если $\operatorname{dim}_{H}(A \times B)<d=\operatorname{dim}_{H} D$, то $A \cap t B=\varnothing$ для почти всех $t \in D$ по $d$-мерной мере Хаусдорфа.

\section{4. Сдвиги и растяжения одной копии самоподобного множества}

Теперь рассмотрим систему сжимающих подобий $\mathcal{F}_{t}=\left\{F_{1}^{t}, \ldots, F_{m}^{t}\right\}$ в $\mathbb{R}^{n}$, зависящую от вещественного или векторного параметра $t \in D$. Пусть $K_{t}$ - аттрактор системы $F_{t}$, а $\pi_{t}: I^{\infty} \rightarrow K_{t}$ - его индексная параметризация. Пусть $i, j \in I$ зафиксированы и $i \neq j$. Пусть только одно отображение $F_{j}^{t}$ системы $\mathcal{F}_{t}$ зависит от параметра $t$, в то время как остальные отображения $F_{k}=F_{k}^{t}$ не зависят. Нас интересует размерность множества

$$
\Delta=\left\{t \in D: F_{i}\left(K_{t}\right) \cap F_{j}^{t}\left(K_{t}\right) \neq \varnothing\right\}
$$

исключительных параметров для двух случаев:

1) $F_{j}^{t}(x)=G(x)+t$, где $t \in \mathbb{R}^{n}$;

2) $F_{j}^{t}(x)=t G(x)+h$, где $t \in(a, b), 0<a<b<1$, а $G$ - изометрия в $\mathbb{R}^{n}$.

Не ограничивая общности, мы можем принять $i=1$ и $j=m$. 
Пусть $\varphi(t, \alpha)=F_{1}\left(\pi_{t}(\alpha)\right), \psi(t, \beta)=F_{m}^{t}\left(\pi_{t}(\beta)\right)$. Для проверки условия (а) теоремы 1 для функций $\varphi$ и $\psi$ нам нужно задать подходящую метрику на индексном пространстве $I^{\infty}$, что позволяет сделать следующая лемма.

Лемма. Пусть $\mathcal{F}=\left\{F_{1}, \ldots, F_{m}\right\}$ - система сжсмающих подобий в $\mathbb{R}^{n}$ с коэффичиентами $\left(p_{1}, \ldots, p_{m}\right)$ и аттрактором $K$. Пусть $\left(q_{1}, \ldots, q_{m}\right) \in(0,1)^{m}-$ фиксированный набор чисел такой, что $p_{i} \leq q_{i}, i \in I$. Пусть $I_{\rho}^{\infty}-$ индексное пространство $I^{\infty}$, наделеннное метрикой $\rho(\alpha, \beta)=q_{\alpha \wedge \beta}$. Тогда:

(а) индексная параметризачия $\pi: I_{\rho}^{\infty} \rightarrow K$ аттрактора системы $\mathcal{F}(\operatorname{diam} K)$-липшицева;

(b) хаусдорфова размерность $\operatorname{dim}_{H} I_{\rho}^{\infty}$ является решением $s$ уравнения $\sum_{i=1}^{m} q_{i}^{s}=1$.

Д о к а з а т е л ь с т в о. Пункт (а) проверяется аналогично случаю [8, Exercise 4.2.4]. Возьмем $\alpha, \beta \in I^{\infty}, \alpha \neq \beta$. Пусть $\boldsymbol{i}=\alpha \wedge \beta$. Тогда $\pi(\alpha)$ и $\pi(\beta)$ оба содержатся во множестве $F_{\boldsymbol{i}}(K)$, диаметр которого равен $p_{\boldsymbol{i}} \cdot \operatorname{diam} K$. Поскольку $p_{\boldsymbol{i}} \leq q_{\boldsymbol{i}}=\rho(\alpha, \beta)$, имеем $\|\pi(\alpha)-\pi(\beta)\| \leq$ $(\operatorname{diam} K) \rho(\alpha, \beta)$, т. е. отображение $\pi$ является $(\operatorname{diam} K)$-липшицевым. Пункт (b) справедлив по теореме [8, Theorem 6.4.3].

Для проверки условия (b) теоремы 1 мы предлагаем следующий метод оценки сверху величины $\left\|\pi_{t}(\alpha)-\pi_{t^{\prime}}(\alpha)\right\|$ через величину $\left\|t-t^{\prime}\right\|$ для всевозможных $\alpha \in I^{\infty}$.

Пусть $V \subseteq \mathbb{R}^{n}$ - такой компакт, что $F_{i}^{t}(V) \subseteq V$ для всех $i \in I, t \in D$. Обозначим $p=$ $\max \left\{p_{1}, \ldots, p_{m-1}, \sup _{t \in D} p_{m}(t)\right\}$ и $\delta\left(t, t^{\prime}\right)=\max _{x \in V, i \in I}\left\|F_{i}^{t}(x)-F_{i}^{t^{\prime}}(x)\right\|$. Тогда по теореме о смещении [6, Theorem 17] для любого $\alpha \in I^{\infty}$ и любых $t, t^{\prime} \in D$ имеем оценку

$$
\left\|\pi_{t}(\alpha)-\pi_{t^{\prime}}(\alpha)\right\| \leq \frac{\delta\left(t, t^{\prime}\right)}{1-p}
$$

Теперь, пользуясь леммой и неравенством (4.1), мы можем применить теорему 1 к разным вариантам поставленной проблемы.

\section{1. Сдвиги}

Теорема 4. Пусть $\mathcal{F}_{t}=\left\{F_{1}, \ldots, F_{m-1}, F_{m}^{t}(x)=G(x)+t\right\}-$ система сжсимающих подобий в $\mathbb{R}^{n}$ с коэфбициентами $\left(p_{1}, \ldots, p_{m}\right)$ и аттрактором $K_{t}$, зависящая от $t \in \mathbb{R}^{n}$. Пусть $p_{1}+p_{m}+\max \left\{p_{1}, \ldots, p_{m}\right\}<1$. Тогда для множества $\Delta=\left\{t \in \mathbb{R}^{n}: F_{1}\left(K_{t}\right) \cap F_{m}^{t}\left(K_{t}\right) \neq \varnothing\right\}$ выполняется неравенство

$$
\operatorname{dim}_{H} \Delta \leq 2 \operatorname{dim}_{S} \mathcal{F}_{0}
$$

Д о к а з а т е л ь с т в о. Пусть $\pi_{t}: I^{\infty} \rightarrow K_{t}$ - индексная параметризация аттрактора системы $\mathcal{F}_{t}$. Обозначим $p_{\max }=\max \left\{p_{1}, \ldots, p_{m}\right\}$. Пусть

$$
\varphi(t, \alpha)=F_{1}\left(\pi_{t}(\alpha)\right), \quad \psi(t, \beta)=G\left(\pi_{t}(\beta)\right)+t, \quad \Phi(t, \alpha, \beta)=\varphi(t, \alpha)-\psi(t, \beta) .
$$

Заметим, что так как коэффициент подобия $p_{m}$ отображения $F_{m}^{t}$ не зависит от параметра $t$, то размерность подобия $\operatorname{dim}_{S} \mathcal{F}_{t}$ системы $\mathcal{F}_{t}$ постоянна и равна $\operatorname{dim}_{S} \mathcal{F}_{0}$. Полагая $q_{i}=p_{i}, i \in I$, из леммы видим, что $\operatorname{dim}_{H} I_{\rho}^{\infty}=\operatorname{dim}_{S} \mathcal{F}_{0}$, а функции $\varphi$ и $\psi$ липшицевы и поэтому удовлетворяют условию (а) теоремы 1.

Проверим условие (b) теоремы 1. Так как при любом выборе ограниченного множества $D \subseteq \mathbb{R}^{n}$ параметров $t$ неравенство (4.1) принимает вид $\left\|\pi_{t^{\prime}}(\alpha)-\pi_{t}(\alpha)\right\| \leq\left\|t^{\prime}-t\right\| /\left(1-p_{\max }\right)$, оно справедливо для всех $t, t^{\prime} \in \mathbb{R}^{n}$. Отсюда получаем

$$
\begin{gathered}
\left\|\varphi\left(t^{\prime}, \alpha\right)-\varphi(t, \alpha)\right\| \leq p_{1}\left\|\pi_{t^{\prime}}(\alpha)-\pi_{t}(\alpha)\right\| \leq \frac{p_{1}\left\|t^{\prime}-t\right\|}{1-p_{\max }} \\
\left\|\psi\left(t^{\prime}, \beta\right)-\psi(t, \beta)\right\| \geq\left\|t^{\prime}-t\right\|-p_{m}\left\|\pi_{t^{\prime}}(\beta)-\pi_{t}(\beta)\right\| \geq \frac{1-p_{\max }-p_{m}}{1-p_{\max }}\left\|t^{\prime}-t\right\| .
\end{gathered}
$$


Наконец, из $\left\|\Phi\left(t^{\prime}, \alpha, \beta\right)-\Phi(t, \alpha, \beta)\right\| \geq\left\|\psi\left(t^{\prime}, \beta\right)-\psi(t, \beta)\right\|-\left\|\varphi\left(t^{\prime}, \alpha\right)-\varphi(t, \alpha)\right\|$ имеем

$$
\left\|\Phi\left(t^{\prime}, \alpha, \beta\right)-\Phi(t, \alpha, \beta)\right\| \geq \frac{1-p_{\max }-p_{m}-p_{1}}{1-p_{\max }}\left\|t^{\prime}-t\right\| .
$$

По условию $p_{1}+p_{m}+p_{\max }<1$, поэтому функции $\varphi$ и $\psi$ удовлетворяют условию (b) теоремы 1. Применяя ее и учитывая, что $\operatorname{dim}_{H}\left(I_{\rho}^{\infty}\right)^{2}=2 \operatorname{dim}_{H} I_{\rho}^{\infty}=2 \operatorname{dim}_{S} \mathcal{F}_{0}$, получаем $\operatorname{dim}_{H} \Delta \leq$ $2 \operatorname{dim}_{S} \mathcal{F}_{0}$.

Следствие 3. В условиях теоремы 4 , если $\operatorname{dim}_{S} \mathcal{F}_{0}<n / 2$, mo $F_{1}\left(K_{t}\right) \cap F_{m}^{t}\left(K_{t}\right)=\varnothing \partial л я$ почти всех $t \in \mathbb{R}^{n}$ по мере Лебега.

В следующем утверждении мы будем подразумевать, что на произведении $\left(\mathbb{R}^{n}\right)^{m}$ задана мера $\mu$, эквивалентная лебеговской мере на $\mathbb{R}^{n m}$.

Следствие 4. Пусть $\mathcal{F}_{\tau}=\left\{F_{1}^{\tau}(x)=G_{1}(x)+t_{1}, \ldots, F_{m}^{\tau}(x)=G_{m}(x)+t_{m}\right\}-$ система сжимающих подобий в $\mathbb{R}^{n}$ с коэфбичиентами $\left(p_{1}, \ldots, p_{m}\right)$ и аттрактором $K_{\tau}$, зависящая от набора параметров $\tau=\left(t_{1}, \ldots, t_{m}\right) \in\left(\mathbb{R}^{n}\right)^{m}$. Пусть $\max \left\{p_{1}, \ldots, p_{m}\right\}<1 / 3 u \operatorname{dim}_{S} \mathcal{F}_{0}<n / 2$. Тогда система $\mathcal{F}_{\tau}$ строго отделима для почти всех $\tau$ по мере $\mu$.

Д о к а з а т е л ь с т в о. Пусть $\pi_{\tau}: I^{\infty} \rightarrow K_{\tau}$ - индексная параметризация аттрактора системы $\mathcal{F}_{\tau}$. Введем отображения $\varphi_{i}(\tau, \alpha)=F_{i}^{\tau}\left(\pi_{\tau}(\alpha)\right), i \in I$.

Рассмотрим множество $\Delta$ всех $\tau \in\left(\mathbb{R}^{n}\right)^{m}$, при которых система $\mathcal{F}_{\tau}$ не удовлетворяет SSC. Ясно, что если $\tau \in \Delta$, то $F_{i}^{\tau}\left(K_{\tau}\right) \cap F_{j}^{\tau}\left(K_{\tau}\right)$ непусто для некоторых $i \neq j$. Поэтому можно представить $\Delta$ в виде объединения

$$
\Delta=\bigcup_{i=1}^{m}\left(\bigcup_{j=1, j \neq i}^{m} \Delta_{i j}\right)
$$

где $\Delta_{i j}=\left\{\tau \in\left(\mathbb{R}^{n}\right)^{m}: F_{i}^{\tau}\left(K_{\tau}\right) \cap F_{j}^{\tau}\left(K_{\tau}\right) \neq \varnothing\right\}, i \neq j$. Заметим, что соотношение $F_{i}^{\tau}\left(K_{\tau}\right) \cap$ $F_{j}^{\tau}\left(K_{\tau}\right) \neq \varnothing$ эквивалентно тому, что $\varphi_{i}(\tau, \alpha)=\varphi_{j}(\tau, \beta)$ для некоторых $\alpha, \beta \in I^{\infty}$. В силу непрерывности функций $\varphi_{k}:\left(\mathbb{R}^{n}\right)^{m} \times I^{\infty} \rightarrow \mathbb{R}^{n}, k \in I$, множества $\widetilde{\Delta_{i j}}$ решений $(\tau, \alpha, \beta)$ уравнений $\varphi_{i}(\tau, \alpha)=\varphi_{j}(\tau, \beta)$ замкнуты. Значит, их проекции $\Delta_{i j}=\operatorname{Pr}_{1}\left(\widetilde{\Delta_{i j}}\right)$ замкнуты в силу замкнутости канонической проекции $\operatorname{Pr}_{1}:\left(\mathbb{R}^{n}\right)^{m} \times I^{\infty} \times I^{\infty} \rightarrow\left(\mathbb{R}^{n}\right)^{m}[9$, Ch. 4, 41, IV, Theorem 1]. Это позволяет нам применить теорему Фубини к характеристическим функциям $\chi_{i j}(\tau)=\chi_{i j}\left(t_{1}, \ldots, t_{m}\right)$ множеств $\Delta_{i j}$ :

$$
M_{i j}=\int_{\mathbb{R}^{n} \times \cdots \times \mathbb{R}^{n}} \cdots \int_{i j} \chi_{i j}(\tau) d t_{1} \cdots d t_{m}=\int_{\mathbb{R}^{n} \times \cdots \times \mathbb{R}^{n}} \cdots \int_{\mathbb{R}^{n}} d t_{1} \cdots d t_{j-1} d t_{j+1} \cdots d t_{m} \chi_{i j}(\tau) d t_{j} .
$$

Так как по условию $p_{i}<1 / 3, i \in I$, и $\operatorname{dim}_{S} \mathcal{F}_{0}<n / 2$, то по следствию 3 при любых фиксированных $t_{k} \in \mathbb{R}^{n}, k \in I, k \neq j$, мы получаем

$$
\int_{\mathbb{R}^{n}} \chi_{i j}(\tau) d t_{j}=0
$$

Значит, $M_{i j}=0$ при $i \neq j$, т. е. множества $\Delta_{i j}$ имеют нулевую $\mu$-меру в пространстве $\left(\mathbb{R}^{n}\right)^{m}$. Таким образом, множество $\Delta$ как конечное объединение множеств нулевой $\mu$-меры также имеет нулевую $\mu$-меру. 


\section{2. Растяжения}

Теорема 5. Пусть $\mathcal{F}_{t}=\left\{F_{1}, \ldots, F_{m-1}, F_{m}^{t}(x)=t G(x)+h\right\}-$ система сжсимающих подобий в $\mathbb{R}^{n}$ с коэффиииентами $\left(p_{1}, \ldots, p_{m-1}, p_{m}(t)\right)$ и аттрактором $K_{t}$, зависящая от параметра $t \in(a, b)$, где $G$ - изометрия в $\mathbb{R}^{n}, 0<a<b<1$. Пусть $r, R>0-$ такие числа, что $r \leq\|G(x)\| \leq R$ для любых $x \in K_{t}, t \in(a, b)$, и пусть $\left(p_{1}+b\right) /\left(1-\max \left\{p_{1}, \ldots, p_{m-1}, b\right\}\right)<r / R$. Тогда для множества $\Delta=\left\{t \in(a, b): F_{1}\left(K_{t}\right) \cap F_{m}^{t}\left(K_{t}\right) \neq \varnothing\right\}$ выполняется неравенство

$$
\operatorname{dim}_{H} \Delta \leq 2 \sup _{t \in(a, b)} \operatorname{dim}_{S} \mathcal{F}_{t}
$$

Д ок а з а т е л ь с т в о. Пусть $\pi_{t}: I^{\infty} \rightarrow K_{t}$ - индексная параметризация аттрактора системы $\mathcal{F}_{t}$. Обозначим $p=\max \left\{p_{1}, \ldots, p_{m-1}, b\right\}$ и $s=\sup _{t \in(a, b)} \operatorname{dim}_{S} \mathcal{F}_{t}$. Пусть

$$
\varphi(t, \alpha)=F_{1}\left(\pi_{t}(\alpha)\right), \quad \psi(t, \beta)=t G\left(\pi_{t}(\beta)\right)+h, \quad \Phi(t, \alpha, \beta)=\varphi(t, \alpha)-\psi(t, \beta) .
$$

Заметим, что $p_{m}(t)=t$, поэтому размерность подобия $\operatorname{dim}_{S} \mathcal{F}_{t}$ системы $\mathcal{F}_{t}$ зависит от параметра $t$. Полагая $q_{i}=p_{i}$ для $1 \leq i<m$ и $q_{m}=b$, из леммы видим, что $\operatorname{dim}_{H} I_{\rho}^{\infty}=s$, a функции $\varphi$ и $\psi$ липшицевы и поэтому удовлетворяют условию (а) теоремы 1.

Проверим условие (b) теоремы 1. Заметим, что $r \leq\left\|G\left(\pi_{t}(\alpha)\right)\right\| \leq R$ для любых $t \in(a, b)$, $\alpha \in I^{\infty}$. Неравенство (4.1) в нашем случае принимает вид $\left\|\pi_{t^{\prime}}(\alpha)-\pi_{t}(\alpha)\right\| \leq R\left|t^{\prime}-t\right| /(1-p)$, откуда

$$
\left\|\varphi\left(t^{\prime}, \alpha\right)-\varphi(t, \alpha)\right\| \leq \frac{p_{1} R\left|t^{\prime}-t\right|}{1-p} ; \quad\left\|t G\left(\pi_{t}(\beta)\right)-t G\left(\pi_{t^{\prime}}(\beta)\right)\right\| \leq \frac{b R\left|t^{\prime}-t\right|}{1-p} .
$$

Из того, что $\left\|\psi\left(t^{\prime}, \beta\right)-\psi(t, \beta)\right\| \geq\left\|t^{\prime} G\left(\pi_{t^{\prime}}(\beta)\right)-t G\left(\pi_{t^{\prime}}(\beta)\right)\right\|-\left\|t G\left(\pi_{t^{\prime}}(\beta)\right)-t G\left(\pi_{t}(\beta)\right)\right\|$, следует

$$
\left\|\psi\left(t^{\prime}, \beta\right)-\psi(t, \beta)\right\| \geq r\left|t^{\prime}-t\right|-\frac{b R\left|t^{\prime}-t\right|}{1-p} .
$$

Наконец, из $\left\|\Phi\left(t^{\prime}, \alpha, \beta\right)-\Phi(t, \alpha, \beta)\right\| \geq\left\|\psi\left(t^{\prime}, \beta\right)-\psi(t, \beta)\right\|-\left\|\varphi\left(t^{\prime}, \alpha\right)-\varphi(t, \alpha)\right\|$ получаем

$$
\left\|\Phi\left(t^{\prime}, \alpha, \beta\right)-\Phi(t, \alpha, \beta)\right\| \geq\left[r-\frac{\left(p_{1}+b\right) R}{1-p}\right]\left|t^{\prime}-t\right| .
$$

По условию $\left(p_{1}+b\right) /(1-p)<r / R$, поэтому функции $\varphi$ и $\psi$ удовлетворяют условию (b) теоремы 1. Применяя ее и учитывая, что $\operatorname{dim}_{H}\left(I_{\rho}^{\infty}\right)^{2}=2 \operatorname{dim}_{H} I_{\rho}^{\infty}=2 s$, получаем $\operatorname{dim}_{H} \Delta \leq 2 s$.

Следствие 5. В условиях теоремъ 5, если $\sup _{t \in(a, b)} \operatorname{dim}_{S} \mathcal{F}_{t}<1 / 2$, mo $F_{1}\left(K_{t}\right) \cap F_{m}^{t}\left(K_{t}\right)=\varnothing$ для почти всех $t \in(a, b)$ по мере Лебега.

Следствие 6. Пусть $\mathcal{F}_{\tau}=\left\{F_{1}^{\tau}(x)=t_{1} G_{1}(x)+h_{1}, \ldots, F_{m}^{\tau}(x)=t_{m} G_{m}(x)+h_{m}\right\}-c u$ стема сжимающих подобий в $\mathbb{R}^{n}$ с аттрактором $K_{\tau}$, зависящая от набора параметров $\tau=\left(t_{1}, \ldots, t_{m}\right) \in(a, b)^{m}$, где $G_{i}, i \in I,-$ изометрии в $\mathbb{R}^{n}, 0<a<b<1$. Пусть $r, R>0-$ такие числа, что $r \leq\left\|G_{i}(x)\right\| \leq R$ для любых $x \in K_{\tau}, \tau \in(a, b)^{m}, i \in I$. Пусть $2 b /(1-b)<r / R$ u $(\ln (1 / m)) / \ln b<1 / 2$. Тогда система $\mathcal{F}_{\tau}$ строго отделима для почти всех $\tau \in(a, b)^{m}$ по мере Лебега.

Д о к а з а т е л ь с т в о. По аналогии с доказательством следствия 4 мы рассматриваем множество $\Delta$ всех $\tau \in(a, b)^{m}$, при которых система $\mathcal{F}_{\tau}$ не удовлетворяет SSC, представляя его в виде объединения

$$
\Delta=\bigcup_{i=1}^{m}\left(\bigcup_{j=1, j \neq i}^{m} \Delta_{i j}\right)
$$


где $\Delta_{i j}=\left\{\tau \in(a, b)^{m}: F_{i}^{\tau}\left(K_{\tau}\right) \cap F_{j}^{\tau}\left(K_{\tau}\right) \neq \varnothing\right\}$, и применяем теорему Фубини к характеристическим функциям $\chi_{i j}(\tau)=\chi_{i j}\left(t_{1}, \ldots, t_{m}\right)$ множеств $\Delta_{i j}, i \neq j$ :

$$
M_{i j}=\int_{(a, b) \times \cdots \times(a, b)} \cdots \int_{(a, b) \times \cdots \times(a, b)} \chi_{i j}(\tau) d t_{1} \cdots d t_{m}=\int_{\cdots} d t_{1} \cdots d t_{j-1} d t_{j+1} \cdots d t_{m} \int_{a}^{b} \chi_{i j}(\tau) d t_{j}
$$

Так как по условию $\left(t_{i}+b\right) /(1-b) \leq 2 b /(1-b)<r / R$ и $\sup _{\tau \in(a, b)^{m}} \operatorname{dim}_{S} \mathcal{F}_{\tau}=(\ln (1 / m)) / \ln b<1 / 2$, то по следствию 5 при любых фиксированных $t_{k} \in(a, b), k \in I, k \neq j$, мы получаем

$$
\int_{a}^{b} \chi_{i j}(\tau) d t_{j}=0
$$

Значит, $M_{i j}=0$, т. е. множества $\Delta_{i j}$ имеют нулевую меру Лебега в $(a, b)^{m}$. Таким образом, множество $\Delta$ как конечное объединение множеств нулевой меры также имеет нулевую меру.

\section{СПИСОК ЛИТЕРАТУРЫ}

1. Hutchinson J. Fractals and self-similarity // Indiana Univ. Math. J. 1981. Vol. 30. no. 5. P. 713-747. doi: 10.1512/iumj.1981.30.30055.

2. Marstrand J. M. Some fundamental geometrical properties of plane sets of fractional dimensions // Proc. Lond. Math. Soc. 1954. Vol. s3-4, iss. 1. P. 257-302. doi: 10.1112/plms/s3-4.1.257.

3. Mattila P. Hausdorff dimension and capacities of intersections of sets in $n$-space // Acta Math. 1984. Vol. 152. P. 77-105. doi: 10.1007/BF02392192.

4. Falconer K. J. Dimensions of intersections and distance sets for polyhedral norms // Real Anal. Exchange. 2004. Vol. 30, no. 2. P. 719-726. doi: 10.14321/realanalexch.30.2.0719.

5. Pollicott M., Simon K. The Hausdorff dimension of $\lambda$-expansions with deleted digits // Trans. Am. Math. Soc. 1995. Vol. 347, no. 3. P. 967-983. doi: 10.2307/2154881.

6. Kamalutdinov K. G., Tetenov A. V. Twofold Cantor sets in $\mathbb{R} / /$ Сиб. электрон. мат. изв. 2018. T. 15. C. 801-814. doi: $10.17377 /$ semi.2018.15.066 .

7. Falconer K. J. Fractal geometry: mathematical foundations and applications. 3rd ed. N Y: J. Wiley and Sons, 2014. 398 p.

8. Edgar G. Measure, Topology, and Fractal Geometry. 2nd ed. New York: Springer-Verlag, 2008. 272 p. doi: 10.1007/978-0-387-74749-1.

9. Kuratowski K. Topology. Vol. 2. N Y; London: Acad. Press, 1968. 608 p.

Поступила 22.03.2019

После доработки 6.05.2019

Камалутдинов Кирилл Глебович

Принята к публикации 13.05.2019

Новосибирский государственный университет

г. Новосибирск

e-mail: kirdan15@mail.ru

\section{REFERENCES}

1. Hutchinson J. Fractals and self-similarity. Indiana Univ. Math. J., 1981, vol. 30, no. 5, pp. 713-747. doi: 10.1512/iumj.1981.30.30055.

2. Marstrand J.M. Some fundamental geometrical properties of plane sets of fractional dimensions. Proc. Lond. Math. Soc., 1954, vol. s3-4, no. 1, pp. 257-302. doi: 10.1112/plms/s3-4.1.257.

3. Mattila P. Hausdorff dimension and capacities of intersections of sets in $n$-space. Acta Math., 1984, vol. 152, pp. 77-105. doi: 10.1007/BF02392192. 
4. Falconer K.J. Dimensions of intersections and distance sets for polyhedral norms. Real Anal. Exchange, 2004, vol. 30, no. 2, pp. 719-726. doi: 10.14321/realanalexch.30.2.0719.

5. Pollicott M., Simon K. The Hausdorff dimension of $\lambda$-expansions with deleted digits. Trans. Am. Math. Soc., 1995, vol. 347, no. 3, pp. 967-983. doi: 10.2307/2154881.

6. Kamalutdinov K.G., Tetenov A.V. Twofold Cantor sets in R. Sib. Elektron. Mat. Izv., 2018, vol. 15, pp. 801-814. doi: 10.17377/semi.2018.15.066.

7. Falconer K.J. Fractal geometry: mathematical foundations and applications. 3rd ed. New York: J. Wiley and Sons, 2014, 398 p. ISBN: 9781118762851.

8. Edgar G. Measure, Topology, and Fractal Geometry. 2nd ed. N Y: Springer-Verlag, 2008,272 p. doi: 10.1007/978-0-387-74749-1.

9. Kuratowski K. Topology. Vol. II. N Y; London: Acad. Press, 1968, 608 p. ISBN: 978-0-12-429202-4 . Translated to Russian under the title Topologiya. T. 2. Moscow, Mir Publ., 1969, 624 p.

Received March 22, 2019

Revised May 6, 2019

Accepted May 13, 2019

Funding Agency: This work was supported by the Russian Foundation for Basic Research (projects no. 19-01-00569, 18-501-51021).

Kirill Glebovich Kamalutdinov, Novosibirsk State University, Novosibirsk, 630090 Russia, e-mail: kirdan15@mail.ru.

Cite this article as: K. G. Kamalutdinov. Self-intersections in parametrized self-similar sets under translations and extensions of copies, Trudy Instituta Matematiki i Mekhaniki URO RAN, 2019, vol. 25, no. 2, pp. 116-124. 